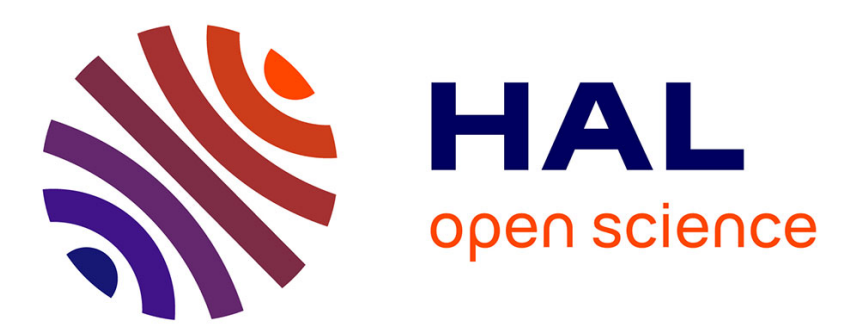

\title{
Recuit pulsé de semiconducteurs par hyperfréquences. Etude de la répartition de la puissance dans l'échantillon
}

\author{
H. Jaouen, P. Chenevier, G. Kamarinos, G. Pananakakis
}

\section{To cite this version:}

H. Jaouen, P. Chenevier, G. Kamarinos, G. Pananakakis. Recuit pulsé de semiconducteurs par hyperfréquences. Etude de la répartition de la puissance dans l'échantillon. Revue de Physique Appliquée, 1984, 19 (4), pp.319-323. 10.1051/rphysap:01984001904031900 . jpa-00245199

\section{HAL Id: jpa-00245199 https://hal.science/jpa-00245199}

Submitted on 1 Jan 1984

HAL is a multi-disciplinary open access archive for the deposit and dissemination of scientific research documents, whether they are published or not. The documents may come from teaching and research institutions in France or abroad, or from public or private research centers.
L'archive ouverte pluridisciplinaire HAL, est destinée au dépôt et à la diffusion de documents scientifiques de niveau recherche, publiés ou non, émanant des établissements d'enseignement et de recherche français ou étrangers, des laboratoires publics ou privés. 
Classification

Physics Abstracts

$78.70 \mathrm{G}-81.40 \mathrm{E}-72.80$

\title{
Recuit pulsé de semiconducteurs par hyperfréquences. Etude de la répartition de la puissance dans l'échantillon
}

\author{
H. Jaouen, P. Chenevier, G. Kamarinos et G. Pananakakis \\ Laboratoire de Physique des Composants à Semiconducteurs $\left({ }^{*}\right)$, \\ ENSERG, 23, rue des Martyrs, 38031 Grenoble Cedex, France \\ (Reçu le 20 septembre 1983, révisé le 5 décembre, accepté le 11 janvier 1984)
}

\begin{abstract}
Résumé. - Les auteurs ont montré récemment la possibilité de recuire par énergie micro-onde pulsée aussi bien du silicium implanté que des couches de silicium polycristallin. Pour des échantillons de haute résistivité il s'est avéré nécessaire d'éclairer simultanément les surfaces traitées en lumière visible. Une simulation détaillée sur calculateur montre qu'en utilisant un appareillage expérimental spécial on obtient une forte absorption de la puissance délivrée par le générateur hyperfréquence sur une large gamme de températures de l'échantillon. Par ailleurs, la stabilité de réglage du montage, ainsi que l'homogénéité du recuit ont été examinées.
\end{abstract}

\begin{abstract}
In recent previous papers we have shown the possibility to anneal implanted monocrystalline as well as polycrystalline silicon by using pulsed microwave energy. For high resistivity samples a simultaneous illumination of the treated surface is needed. A detailed computer simulation shows that using a special experimental bench almost the total power delivered by the microwave generator is absorbed into the silicon for a very large range of temperature. Besides, the adjustement stability of the apparatus as well as the annealing homogeneity have been examined.
\end{abstract}

\section{Introduction.}

Plusieurs méthodes de recuit pulsé destinées à être utilisées dans les filières de fabrication des circuits intégrés et des cellules solaires sont à l'étude depuis quelques années dans de nombreux laboratoires : recuits par laser, par lampe à arc, par lampe flash. par faisceau d'électrons, par rayonnement $\mathrm{X}$, par rayonnement thermique $[1,2]$.

Dans des publications récentes $[3,4]$ nous avons montré la possibilité de recuire un semiconducteur en utilisant l'énergie micro-onde pulsée. Cette nouvelle méthode a été testée aussi bien sur du silicium monocristallin endommagé par implantation que sur une couche de silicium polycristallin déposé [5]. De plus, pour les couches de résistivité élevée un éclairage simultané en lumière visible a été nécessaire pour amorcer le recuit.

La valorisation de ce nouveau procédé de recuit et son éventuelle application dans des conditions industrielles nécessitent des réponses à trois questions essentielles :

1) Quelle est l'influence des paramètres électriques de la plaquette à recuire sur le réglage de l'appareil ? En effet, l'emploi industriel d'une machine à micro-

(*) ERA CNRS No 659 . onde exige un réglage définitif ne dépendant pas de manière critique, par exemple, de la conductivité originelle et de sa variation avec la température.

2) Quelle est l'homogénéité du recuit et comment dépend-elle des conditions de dépôt de la puissance?

3) Quel est le coût d'une machine industrielle ?

Dans le présent article, nous effectuons un travail de simulation qui nous permet d'aborder certains aspects des questions 1) et 2) pour le montage utilisé lors des essais de faisabilité, avec un échantillon placé dans une cavité dérivée d'un té magique et fonctionnant en bande $\mathrm{X}$ [3].

En particulier, nous examinons l'homogénéité du dépôt de puissance et la possibilité d'avoir un bon transfert de puissance dans la plaquette, pour une large gamme de conductivité et par conséquent de température, avec des réglages fixes.

En ce qui concerne la troisième question, les puissances mises en jeu permettent d'utiliser des générateurs à fréquence industrielle $(2,45 \mathrm{GHz})$ standards d'un coût non prohibitif.

\section{Détermination des conditions d'un réglage fixe.}

Le chauffage par énergie micro-onde d'une plaquette de silicium est dû à la dissipation par effet Joule des courants induits. Pour effectuer un recuit avec un bon 
rendement énergétique il faut donc que le coefficient de transfert $\eta$ du montage utilisé (défini comme étant le rapport de la puissance dissipée par effet Joule dans l'échantillon, à la puissance émise par le générateur) ait une valeur proche de l'unité.

En supposant que l'échantillon est porté à une température uniforme $T$, nous pouvons calculer $\eta$ en fonction de la conductivité $\sigma$ du semiconducteur. De plus, d'après les données de la littérature [6], on peut exprimer, dans le cas du silicium, $\sigma$ en fonction de la température. Nous l'avons fait pour un échantillon de conductivité égale à $12,5 \Omega^{-1} \mathrm{~m}^{-1}$ $(\rho=8 \Omega \mathrm{cm})$ à la température ambiante afin de connaître $\eta$ à diverses températures. La loi $\sigma(T)$ d'un échantillon ayant, à la température ambiante, une résistivité différente n'est pas modifiée sensiblement à haute température, ce qui permet d'étendre la validité des commentaires qui seront faits par la suite, à tout échantillon.

Avant d'étudier le montage utilisé lors des essais de faisabilité comme il a été dit plus haut, nous avons envisagé le montage le plus simple dans lequel la plaquette ferme complètement le guide de sortie du générateur. Nous avons par la suite calculé le coefficient $\eta$ de ce montage en fermant le guide derrière l'échantillon, soit par un court-circuit, soit par une charge adaptée. La'figure 1 (courbe a) montre alors la variation de $\eta$ en fonction de $\sigma$ et de $T$ obtenus avec une charge adaptée. Dans le cas de l'utilisation d'un court-circuit, les valeurs de $\eta$ sont beaucoup plus faibles.

Pour tous les cas étudiés dans cet article, nous avons considéré un guide rectangulaire RG52/U fonctionnant en mode $H_{10}$ et une plaquette rectangulaire de silicium de dimensions $10,2 \mathrm{~mm} \times 22,9 \mathrm{~mm}$ et de $300 \mu \mathrm{m}$ d'épaisseur.

En revenant sur la courbe a de la figure 1, nous remarquons qu'à $900^{\circ} \mathrm{C}$, température du recuit, $\eta$ est très faible (environ $3 \%$ ) et que sa valeur maximale est de l'ordre de $45 \%$ à $300{ }^{\circ} \mathrm{C}$ environ. La décroissance observée de $\eta$ avec $\sigma$ ou $T$ est due au comportement métallique de l'échantillon, à haute température. Il est à remarquer qu'à cette température la terminaison du guide ne joue aucun rôle, $\eta$ reste petit quelle que soit la terminaison. Par conséquent, il est très difficile d'obtenir un recuit par énergie micro-onde avec ce type de montage.

C'est la raison pour laquelle nous avons adopté le montage utilisé lors des essais de faisabilité qui comporte une cavité résonnante, dérivée d'un té magique. Le résonateur, alimenté par le bras (3), comporte deux bras (1) (2) fermés par des pistons de courtcircuit mobiles permettant d'agir sur la fréquence de résonance et le coefficient de couplage, le bras (4) étant fermé par l'échantillon (Fig. 2).

Le silicium étant pratiquement transparent aux hyperfréquences à basse température pour les épaisseurs standards des plaquettes, le bras (4) contenant l'échantillon doit être fermé sur une charge ou un

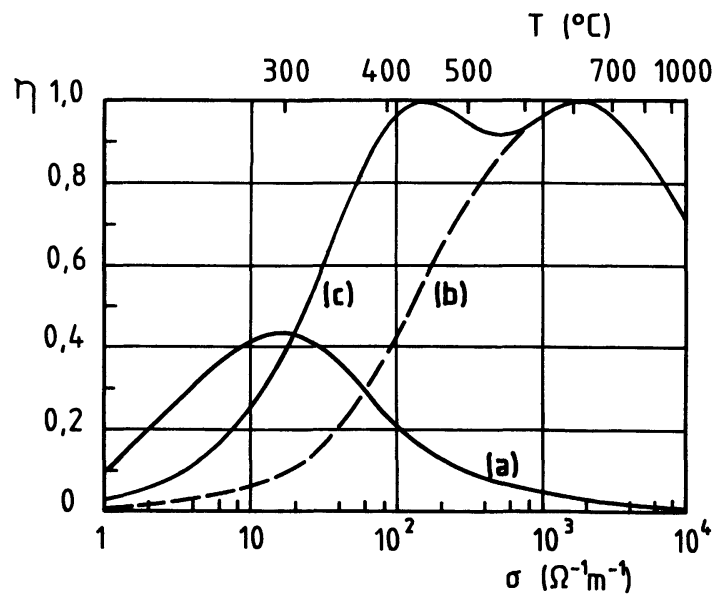

Fig. 1. - Evolution du coefficient de transfert $\eta$ à $10 \mathrm{GHz}$, pour une plaquette de silicium de $300 \mu \mathrm{m}$ d'épaisseur en fonction de la conductivité et de la température. Courbe a : plaquette fermant un guide RG52/U. Courbe $\mathrm{b}:$ montage de la figure $2 \mathrm{a}(x=0,9821 ; y=0,9998)$. Courbe $\mathrm{c}:$ montage de la figure $2 \mathrm{~b}(x=0,9821 ; y=0,9998 ; z=0,4932)$.

[Ratio $\eta$ as a function of sample conductivity and temperature (for a $300 \mu \mathrm{m}$ thick silicon wafer at $10 \mathrm{GHz}$ ). Curve a : with a wafer closing the RG52/U waveguide; curve b : with the set up of figure $2 \mathrm{a}(x=0.9821, y=0.9998)$. Curve $\mathrm{c}$ : with the set up of figure $2 \mathrm{~b}(x=0.9821, y=0.9998$, $z=0.4932)$.]

piston de court-circuit mobile supplémentaire. Ces deux possibilités ont été ětudiées et simulées afin d'obtenir une valeur de $\eta$ proche de l'unité pour une large gamme de température, en optimisant la position des pistons de court-circuit du montage (Figs. 2a et $2 b$ respectivement).

Dans le cas de la terminaison par une charge adaptée cette optimisation a été réalisée en introduisant deux variables réduites $x, y$ définies par les relations $x=\cos \left[\beta_{\mathrm{g}_{0}}\left(l_{1}-l_{2}\right)\right]$ et $y=\cos \left[\beta_{\mathrm{go}_{0}}\left(l_{1}+l_{2}\right)\right]$; $l_{1}, l_{2}$ sont les distances séparant les plans de référence du té magique et les plans de court-circuit dans les bras respectifs et $\beta_{\mathrm{go}}$ est la constante de propagation dans les guides. La variable y rend compte du " volume " de la cavité par le biais de la somme $l_{1}+l_{2}$, $x$ représentant l'" excentricité " par la différence $l_{1}-l_{2}$. Notre étude montre qu'un tel montage ne permet d'obtenir des valeurs de $\eta$ proches de 1 que dans une plage étroite de température, située au voisinage de la température du recuit. A la température ambiante $\eta$ est inférieur à $10 \%$, le rendement énergétique étant médiocre pour les températures moyennes (Fig. 1 courbe b).

Dans le cas où la terminaison du bras (4) de la cavité est faite avec un piston de court-circuit mobile situé à la distance $l_{4}$ de la face arrière de l'échantillon, on introduit un paramètre de réglage supplémentaire pour les températures moyennes et faibles, $z$, exprimé par la relation : $z=l_{4}\left(\beta_{\mathrm{g}_{0}} / 2 \pi\right)$. En effet, en réglant les pistons (1) et (2) de telle manière que l'on ait $\eta=1$ 


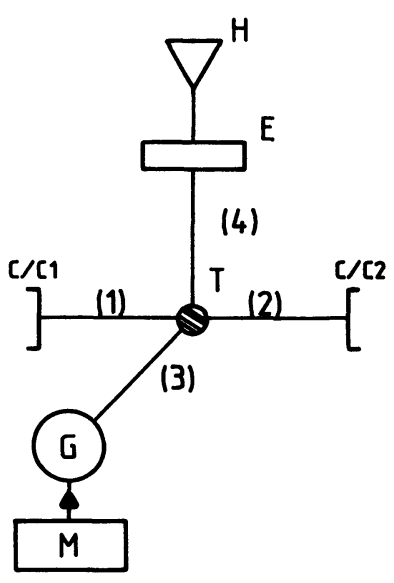

(a)

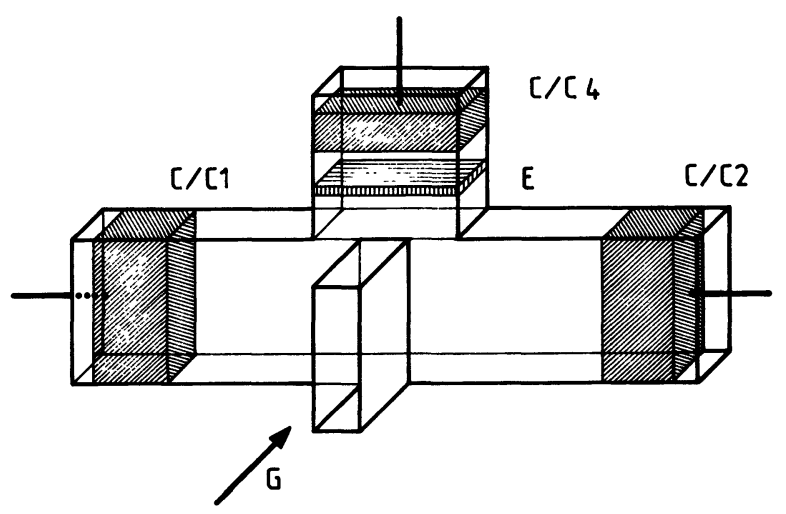

(c)

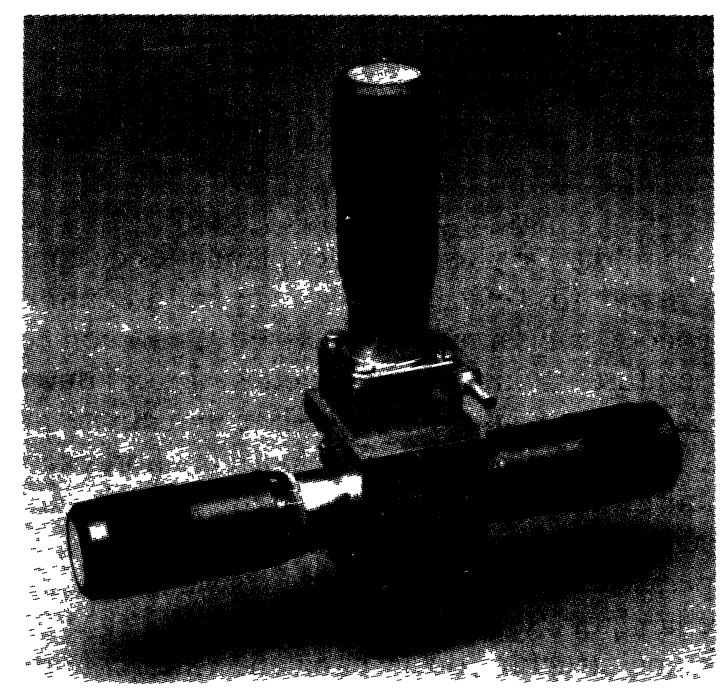

Fig. 2. - Montage expérimental. M : Modulateur; G : Générateur; $\mathrm{T}$ : Té magique; $\mathrm{C} / \mathrm{C}$ : piston de court-circuit ; $\mathrm{E}$ : porte-échantillon. (a) Utilisation d'une charge adaptée placée derrière l'échantillon, (b) et (c) utilisation d'un piston de court-circuit de compensation.

[Experimental set up : modulator (M), generator (G), magic $T(T)$, mobile short-circuit termination $(C / C)$, sample holder (E). (a) with a matched load termination, (b) and (c) with a mobile short-circuit termination.] à haute température (couplage critique), le réglage du piston de court-circuit du bras (4) peut se faire de manière à obtenir des conditions proches du couplage critique aux températures moyennes (Fig. 1, courbe c). Nous arrivons ainsi à absorber plus de $90 \%$ de la puissance incidente dans le silicium entre $300^{\circ} \mathrm{C}$ et $900^{\circ} \mathrm{C}$ et environ $35 \%$ à température ambiante. Si le besoin se présente, l'amorçage du recuit à cette température peut être facilité par l'éclairage simultané de la plaquette en lumière visible.

\section{Répartition de la puissance dissipée dans l'échan- tillon.}

Dans le cas des montages étudiés précédemment, la densité de puissance (par unité de surface) sur une section droite du guide n'est pas constante. Pour le mode de fonctionnement étudié $\left(\mathrm{H}_{10}\right)$, on sait que cette densité reste constante lorsqu'on se déplace parallèlement à la petite face du guide $(Y)$ et qu'elle varie, lorsqu'on se déplace parallèlement à la grande face du guide $(X)$ en $\sin ^{2} X$. Dans la plaquette de silicium considérée, la puissance est donc essentiellement dissipée dans une bande centrée au milieu de la grande dimension du guide. La répartition de la température dans le plan $X Y$ est bien évidemment déterminée par le bilan entre la puissance déposée et les fuites de chaleur dues au contact existant entre la plaquette et le guide métallique.

Dans notre simulation, nous n'avons pas résolu l'équation de diffusion de la chaleur mais nous avons supposé (comme il a été dit plus haut, section 2) que l'échantillon est porté à une température uniforme, ce qui correspond au cas idéal d'un échantillon thermiquement isolé. Dans le cas contraire, la température de l'échantillon est non uniforme, ce qui est à l'origine des contraintes mécaniques observées expérimentalement lors du recuit [7].

Afin d'éviter les problèmes posés par cette nonuniformité de la température, nous avons étudié un autre montage hyperfréquence utilisant un résonateur en anneau, qui est en cours de réalisation (Fig. 3). Ce montage améliorera d'une part, l'uniformité du dépôt de puissance et il assurera un bon isolement thermique entre la plaquette et les parois du résonateur, d'autre part. Un recuit de la plaquette à température uniforme et en l'absence de contraintes pourra donc être réalisé.

En ce qui concerne la répartition de la puissance dans le sens de la profondeur de la plaquette les résultats de la simulation exposée en section 2 sont représentés figure 4. Nous remarquons que si l'échantillon est irradié à haute température (ce qui correspond à une conductivité élevée) la dissipation de la puissance se fait sur la face avant de la plaquette. Compte tenu du comportement métallique du silicium à ces températures, l'effet de peau est manifeste. $\mathrm{Si}$, par contre, l'irradiation a lieu à basse température (faible valeur de $\sigma$ ) la dissipation ne se fera que si le 

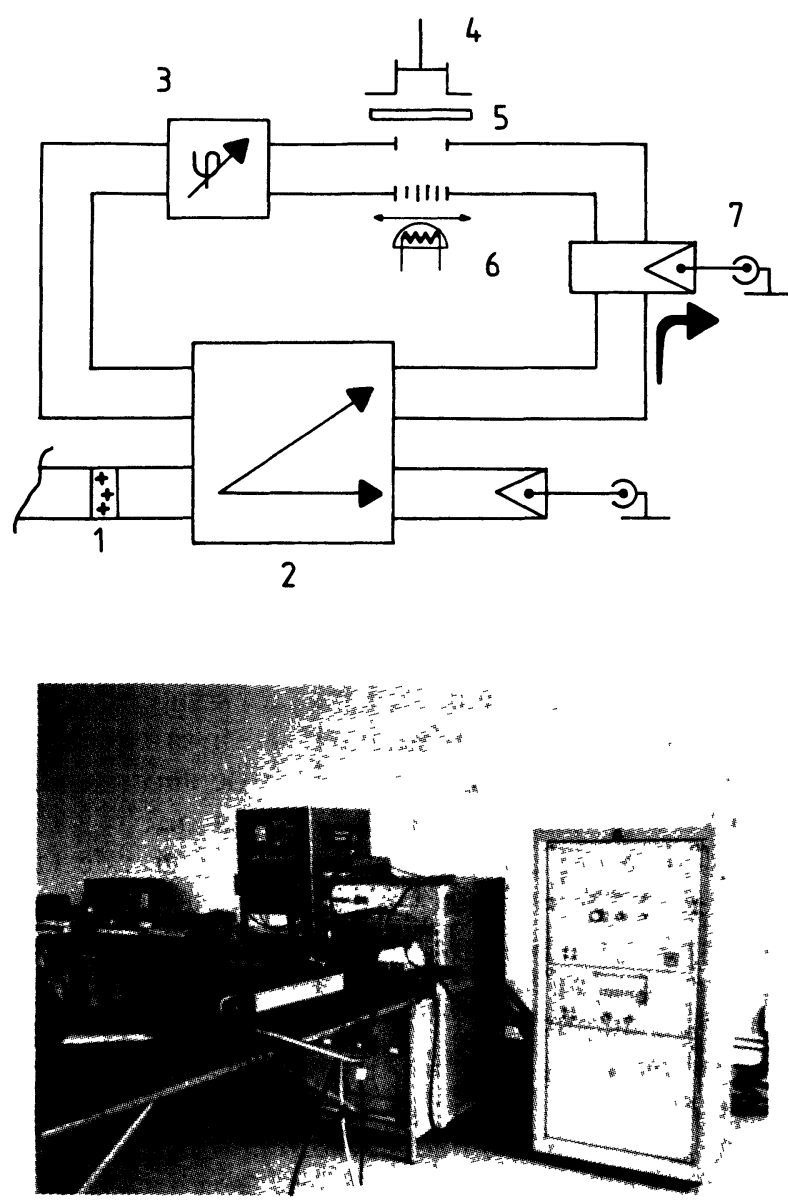

Fig. 3. - Utilisation d'un résonateur en anneau 1 : fenêtre de pressurisation ; 2 : coupleur directif à couplage variable permettant d'ajuster la puissance induite dans l'anneau; 3 : déphaseur assurant l'accord en fréquence; $4:$ piston de court-circuit de compensation; 5 : échantillon isolé thermiquement par utilisation de pièges à ondes; $6:$ système d'éclairement de l'échantillon; $7:$ mesure de la puissance induite dans l'anneau.

[Use of a ring resonator : (1) pressurization window, (2) directional coupler, (3) phase shifter, (4) mobile short circuit termination, (5) thermally isolated sample holder, (6) sample illumination set up, (7) measurement of the induced power in the ring.]

réglage du piston de court-circuit du bras (4) est optimum, et elle aura lieu près de la face arrière de la plaquette. Dans tous les cas, vu le temps d'exposition aux hyperfréquences (de l'ordre de $100 \mathrm{~ms}$ ) et l'épaisseur de l'échantillon étudié $(300 \mu \mathrm{m})$ il ne doit pas y avoir de gradient de température dans le sens de l'épaisseur.

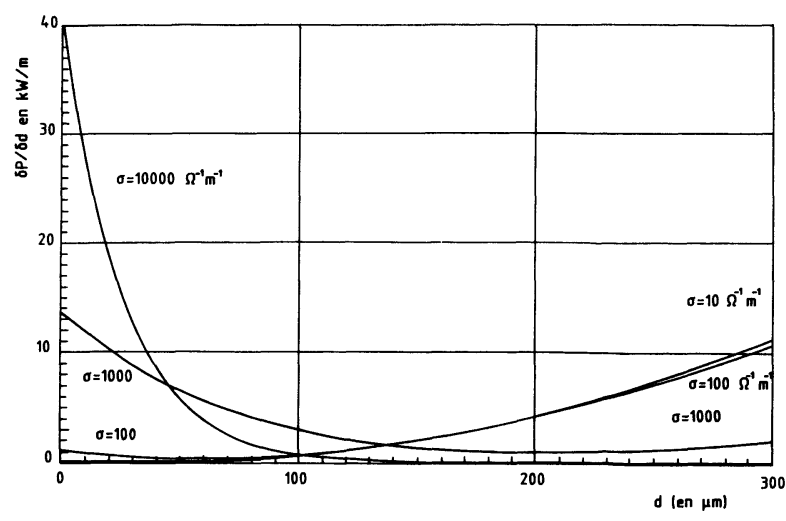

Fig. 4. - Gradient de la puissance dissipée dans une plaquette de silicium de $300 \mu \mathrm{m}$ d'épaisseur en fonction de la profondeur $d$ dans la plaquette (montage de la figure 2; $x=0,9821 ; y=0,9998 ; z=0,4932$ ).

[Dissipated power gradient in a $300 \mu \mathrm{m}$ thick silicon wafer as a function of the depth $d$ (set up of figure $2 \mathrm{~b} ; x=0.9821$, $y=0.9998, z=0.4932)$.]

\section{Conclusion.}

Nous avons montré qu'avec une structure résonnante il était possible d'avoir une bonne efficacité de dépôt de puissance dans le silicium thermiquement isolé. Le réglage du montage est réalisé avec trois pistons un bon transfert de puissance en haute température. Le troisième, situé derrière l'échantillon, peut améliorer le rendement en basses et moyennes températures et assurer ainsi de bonnes conditions d'amorçage du recuit.

Ce réglage peut être fixe dans une gamme étendue de dopage des échantillons et par conséquent le montage n'a pas à être réajusté quand on change d'échantillon. De plus, pour des plaquettes de conductivité standard, l'efficacité à la température ambiante est encore suffisante pour permettre la montée en température. Pour des plaquettes de conductivité très faible on peut augmenter la conductivité apparente initiale par génération de porteurs libres supplémentaires en éclairant la plaquette [5]. En ce qui concerne l'homogénéité du recuit, et donc de la température, il faut augmenter la résistance thermique entre la plaquette et les parois de la cavité et se placer dans une structure où la circulation des courants à la surface du silicium soit aussi homogène que possible. C'est ce que nous espérons obtenir avec un anneau résonnant en cours de réalisation. 


\section{Bibliographie}

[1] SifFERT, P., Recuit par faisceaux d'énergie, rapport GCIS 1983, CRN Phase, Strasbourg.

[2] Appleton, B. R., Celler, G. K., Laser and ElectronBeam Interactions with solids (North-Holland, New York) 1982, V4.

[3] Chenevier, P., Cohen, J., Kamarinos, G., J. Physique Lett. 43 (1982) L-291.

[4] Chenevier, P., Cohen, J., Kamarinos, G., Proceed. 4th EC Photovoltaic solar energy conf., Stresa (D. Reidel) 1982, 1065.
[5] Chenevier, P., Cohen, J., Kamarinos, G., PananaKakIs, G., JaOuen, H., Proceed. Melecon' 83, Athènes (E. N. Protonotarios, G. I. Stassinopoulos, P. P. Civalleri) 1983, D8.07.

[6] Morin, F. J. and Maita, J. P., Phys. Rev. 96 n $^{\circ} 1$ (1954) 28.

[7] Jaouen, H., Cohen, J., Chenevier, P., Kamarinos, G., PananaKakis, G., Recuit pulsé par énergie microonde, rapport GCIS 1982, LPCS Grenoble. 